\title{
6 Extraktive Enklaven und frontiers in Afrika
}

Im Rahmen des SFB 1199 betrachten wir den rezenten industriellen Goldboom in Afrika unter der Fragestellung, ob die weltweit steigende Nachfrage nach Rohstoffen bestimmte Raumformate begünstigt. ${ }^{1}$ Im Zentrum steht dabei die Frage, ob die extraktive Enklave ein solches Raumformat darstellt. Wir beziehen uns dabei auf die eingangs genannte These von James Ferguson. ${ }^{2}$ Er postulierte, dass sich im Zuge eines globalen „extraktiven Neoliberalismus“ Fördergebiete für metallische oder mineralische Rohstoffe in Afrika durch einen Prozess der Einschließung (enclaving) der Offshore-Ölförderung angleichen. Das Modell dafür sei der angolanische Staat, wo die politische Elite mit Unterstützung internationaler Banken, Unternehmen und Sicherheitsfirmen den Ölsektor erfolgreich vom Rest der Wirtschaft abgekoppelt habe, um sich Gewinne privat aneignen zu können. Anders, als es das Bild globaler „Kapitalströme“ suggeriere, „hüpfe“ globales Kapital von einer extraktiven Enklave zur anderen ${ }^{3}$. Extraktive Enklaven seien stärker mit Zentren der globalen Wirtschaft verbunden als mit den Ländern, in denen sie sich etablieren. Dies begünstige unterschiedliche raumbezogene Formen von Regierungsführung (governance): Während extraktive Enklaven mit Duldung oder Unterstützung des sie umgebenden Nationalstaats gesetzlich oder physisch abgegrenzt und von privaten Sicherheitsfirmen bewacht werden, wird die Verwaltung der Räume zwischen Enklaven zunehmend nicht-staatlichen Organisationen übertragen oder vernachlässigt. Dies trage dazu bei, dass entgegen dem von internationalen Finanz- und Entwicklungsorganisationen behaupteten kausalen Zusammenhang zwischen guter Regierungsführung (good governance) und wirtschaftlicher Entwicklung es oft jene Staaten mit einer eklatant schlechten Regierungsführung sind, die das meiste globale Kapital anziehen und die höchsten Wachstumsraten vorweisen können.

1 Teilprojekt B06 „Goldbergbau und Neuregulierungen (sub)nationaler Räume in Afrika“ mit Diana Ayeh und Kai Roder. Siehe auch die parallele Studie von Arne Harms zu Kohlenstoffspeichern als „akkretiven Enklaven“ im Kontext von Maßnahmen gegen den Klimawandel: A. Harms, Accretive Enclaves: Carbon Sequestration and Market-based Conservation in India, Working Paper Series SFB 1199 No. 12, Leipzig 2018, https://research.uni-leipzig.de/ sfb1199/wp-content/ uploads/2018/10/WP12_Harms.pdf (zuletzt aufgerufen 25.4.2019)

2 J. Ferguson, „Seeing Like an Oil Company: Space, Security, and Global Capital in Neoliberal Africa“, American Anthropologist 107 (2005) 3, S. 377-382; J. Ferguson, „Governing Extraction. New Spatializations of Order and Disorder in Neoliberal Africa“, in: J. Ferguson, Global Shadows. Africa in the Neoliberal World Order, Durham und London: Duke University Press, 2006, S. $194-$ 210.

3 Ferguson, „Seeing Like an Oil Company“, S. 380.

Ә OpenAccess. 1C 2020, Katja Werthmann, published by De Gruyter. (cc) BY-NC-ND This work is licensed under the Creative Commons Attribution-NonCommercial-NoDerivatives 4.0 License https://doi.org/10.1515/9783110645996-006 
Ferguson kontrastiert diese neuen, sozial „dünnen“ extraktiven Enklaven in Afrika mit früheren, sozial „dichten“ Formen von industriellem Bergbau am Beispiel des kolonialen und nachkolonialen Kupferbergbaus in Sambia, wo Bergarbeiter und ihre Familien in eine paternalistische Unternehmensstruktur und -kultur eingebunden waren. Dieses „dichte“ Modell endete in den 1980er Jahren durch den Verfall von Rohstoffpreisen, Korruption, Missmanagement und gewaltsame Konflikte. Arbeitslos gewordene Bergleute und andere Mitarbeiter von Minenunternehmen waren nun auf sich selbst gestellt. Viele hatten größte Schwierigkeiten damit, in der informellen urbanen Wirtschaft oder durch eine „Rückkehr“ aufs Land wieder Fuß zu fassen. ${ }^{4}$ Es gilt allerdings zu berücksichtigen, dass der sambische Kupferbergbau einen Sonderfall darstellte und sich ein solches „sozial dichtes“ System in anderen Bergbaugebieten Afrikas nicht herausbildete. ${ }^{5}$

Ferguson schließt: „it is worth asking whether Africa's combination of privately secured mineral-extraction enclaves and weakly governed humanitarian hinterlands might constitute not a lamentably immature form of globalization but, rather, a quite ,advanced' and sophisticated mutation of it. If so, the forms of ,global economy 'that have developed in some mineral-rich African countries in recent years might show us not only a theoretically interesting anomaly but also a frightening sort of political-economic model for some other regions that combine mineral wealth with political intractability“ ${ }^{6}$

Fergusons These wurde von mehreren Autoren auf verschiedene Fallbeispiele angewandt und modifiziert. Am Beispiel von Katanga in der Demokratischen Republik Kongo beschreibt Jana Hönke das Entstehen von „Festungen der Pro-

4 J. Ferguson, Expectations of Modernity. Myths and Meanings of Urban Life on the Zambian Copperbelt, Berkeley u. a.: University of California Press, 1999; T. Makori, „Abjects retraités, jeunesse piégée: récits du déclin et d'une temporalité multiple parmi les générations de la ,Copperbelt" congolaise“, Politique africaine 131 (2013) 3, S. 51-73; B. Rubbers, Le paternalisme en question. Les anciens ouvriers de la Gécamines face à la libéralisation du secteur minier katangais (R. D. Congo), Paris: L'Harmattan, 2013.

5 R. E. Dumett, El Dorado in West Africa. The Gold-Mining Frontier, African Labor, and Colonial Capitalism in the Gold Coast, 1875-1900, Athens/Oxford: Ohio University Press, James Currey, 1998; J. Hönke, „Extractive Orders. Transnational Mining Companies in the Nineteenth and Twenty-First Centuries in the Central African Copperbelt“, in: R. Southall und H. Melber (Hg.), A New Scramble for Africa. Imperialism, Investment and Development, Scottsville: University of KwaZulu-Natal Press, 2009, S. 274-298; C. Mark-Thiesen, Mediators, Contract Men, and Colonial Capital. Mechanized Gold Mining in the Gold Coast Colony, 1879-1909, Rochester: University of Rochester Press, 2018.

6 Ferguson, „Seeing Like an Oil Company“, S. 380. 
duktion“. ${ }^{7}$ Diese Festungen umgeben sich jedoch mit einem durchlässigen und flexiblen „Schutzgürtel“, innerhalb dessen sie selektiv mit verschiedenen lokalen und translokalen Akteuren interagieren. Hönke betont, dass private Firmen gegenüber dem Staat nicht völlig autonom werden. Es entstünden vielmehr komplexe Geflechte und „Knotenpunkte“ der Rohstoff-Verwaltung, in welche nicht nur Regierungen und Unternehmen, sondern auch internationale und nationale Nichtregierungsorganisationen, Aktivistengruppen oder Lokalpolitiker involviert seien, die sich wiederum auf unterschiedliche Normen, Diskurse und Rollenmodelle beziehen.

Hannah Appel illustriert die These Fergusons mit dem Beispiel der Ölförderung in Äquatorialguinea. Mittels einer Rhetorik der Entflechtung (disentanglement) verleugnet dort die amerikanische Ölfirma ihre tatsächliche wirtschaftliche und politische Verflechtung mit dem äquatorialguineischen Staat. Die räumliche und diskursive Separation der Ölfirma vom Staat ermöglicht es den Mitarbeitern der Firma, Armut, Umweltschäden und Korruption „dort draußen“ zu beklagen, als wären sie nicht selbst integral in deren Fortbestehen verstrickt. Dies erfordert ständige „Distanzarbeit“ (distancing work), z. B. in Form hochritualisierter Sicherheitsroutinen. ${ }^{8}$

Die Entstehung extraktiver Enklaven hat unmittelbare räumliche Effekte wie z. B. Landschaftswandel, Baumaßnahmen und damit einhergehende sozialräumliche Segregation. Appel kontrastiert am Beispiel der Ölförderenklave in Äquatorial-Guinea zwei unterschiedliche urbane Räume: einerseits die luxuriöse, abgeschottete Wohnsiedlung für das amerikanische Personal der Ölfirma und andererseits die benachbarte Hauptstadt Malabo, in der es trotz der Öleinnahmen an grundlegender Infrastruktur mangelt. Sowohl der Staat als auch die Ölfirma lehnen die Verantwortung für die öffentliche Infrastruktur ab. Gleichzeitig fordert der Staat von der Ölfirma, Prestigebauten zu errichten, deren einzige Funktion die Symbolisierung von „sichtbarem Investment“ ist. $^{9}$

Entgegen der Annahme, das Entstehen von extraktiven Enklaven verringere automatisch die Souveränität von Nationalstaaten, verweisen Emel et al. mit

7 J. Hönke, Transnational Pockets of Territoriality. Governing the Security of Extraction in Katanga (DRC). Working Paper Series of the Graduate Centre Humanities and Social Sciences of the Research Academy Leipzig 2 (2009).

8 H. C. Appel, „Offshore Work: Oil, Modularity, and the How of Capitalism in Equatorial Guinea“, American Ethnologist 39 (2012) 4, S. 692-709, hier S. 706.

9 H. C. Appel, „Walls and White Elephants: Oil Extraction, Responsibility, and Infrastructural Violence in Equatorial Guinea“, Ethnography 13 (2012) 4, S. 439-465. 
Bezug auf Agnews ${ }^{10}$ „Regime der Souveränität“ darauf, dass Nationalstaaten und multinationale Unternehmen interdependente Akteure sind. ${ }^{11}$ Die Prozesse der Aushandlung von „Rohstoff-Souveränität“ (resource sovereignty) in Tansania resultieren in einer Form der „Staat-Kapital-Souveränität“ als Konstellation von sich gegenseitig verstärkenden Bedingungen und Zwängen, welche der Staat Unternehmen auferlegt und umgekehrt.

Ähnlich argumentieren Muriel Côte und Benedikt Korf mit Bezug auf die Goldförderung in Burkina Faso, dass es hier zu einer „Koproduktion“ von Regulierung zwischen Staat und nicht-staatlichen Akteuren bzw. zu einer „Plurifizierung “ regulativer Autorität komme. ${ }^{12}$ Industrieller, formeller und nicht-industrieller, informeller Bergbau überlappen in ihrem Beispiel aus dem Norden des Landes auf demselben Gebiet. In den Aushandlungen über die Tolerierung von nicht-industriellem Bergbau auf einem konzessionierten Bergwerk kommen unterschiedliche Interessen und Legitimitäten zum Tragen, wobei das ausländische Unternehmen nicht immer am längeren Hebel sitzt. Die Persistenz des nicht-industriellen Bergbaus führt zu neuen Formen von Regulierung seitens des Staates in Form neuer Institutionen für Ressourcenverwaltung und Sicherheit. Gleichzeitig etablieren sich Akteure des nicht-industriellen Bergbaus durch Kommunalwahlen als Lokalpolitiker und verstärken dadurch die Bedeutung des Staates. So erlange der Staat „durch die Hintertür“ Zugang zu einer extraktiven Enklave.

Géraud Magrin vergleicht die extraktive Enklave mit einer Zwiebel: jede Lage enthalte eine weitere Form von Kontrolle und Zwang. ${ }^{13}$ Eine Form von Unterenklave sind beispielsweise die Unterkünfte für weiße Mitarbeiter in einem Holzfäller-Camp in der Demokratischen Republik Kongo, die von den Unterkünften schwarzer Mitarbeiter getrennt sind. ${ }^{14}$ Kontrollen seien dort besonders strikt, wo ein Rohstoff leicht gestohlen werden könne. Andererseits könnten die von Bergbauunternehmen errichteten Infrastrukturen wie Straßen, Brücken oder Tele-

10 J. Agnew, „Sovereignty Regimes: Territoriality and State Authority in Contemporary World Politics“, Annals of the Association of American Geographers 95 (2005) 2, S. 437-461; Ders., Globalization and Sovereignty, Lanham MD, 2009.

11 J. Emel, M. T. Huber und M. H. Makene, „Extracting Sovereignty: Capital, Territory, and Gold Mining in Tanzania“, Political Geography 30 (2011), S. 70 - 79.

12 M. Côte und B. Korf, „Making Concessions: Extractive Enclaves, Entangled Capitalism and Regulative Pluralism at the Gold Mining Frontier in Burkina Faso“, World Development 101 (2018), S. $466-476$.

13 G. Magrin, Voyage en Afrique rentière, Paris: Publications de la Sorbonne, 2013, S. 225.

14 T. Hendriks, „A Darker Shade of White: Expat Self-Making in a Congolese Rainforest Enclave“, Africa 87 (2017) 4, S. 683-701. 
fonmasten auf lokaler Ebene zu einer Auflösung von Isolation (désenclavement) führen. ${ }^{15}$

Benjamin Rubbers zeigt am Beispiel von Arbeitersiedlungen im kongolesischen Kupfergürtel, dass Bergbau unterschiedliche Arten von Räumen hervorbringt. ${ }^{16}$ Diesen Prozess prägen wiederum über verschiedene historische Phasen hinweg unterschiedliche Akteure, Machtstrategien und staatliche Regulierungsbemühungen, die sich nicht alle unter das „Dampfwalzenkonzept“ der Enklave subsumieren ließen. Unter anderem könne das Konzept historischen Wandel und regionale Unterschiede nicht erfassen.

Auch nicht-industrieller Bergbau kann Enklaven hervorbringen. Jean Omasombo Tshonda beschrieb Diamanten-Schürfgebiete in abgelegenen Gegenden Zaïres (heute Demokratische Republik Kongo), die sich zu „Staaten im Staat“ entwickeln konnten. ${ }^{17}$ Gewaltunternehmer etablierten dort Lager, welche Miniaturversionen des Mobutu-Staates glichen. Der Zugang wurde von „Wachtmeistern“ kontrolliert, die Händlern „Aufenthaltsgenehmigungen“ erteilten, nachdem diese eine Art Zollgebühr entrichtet hatten. Die Lager waren in Sektionen unterteilt, die jeweils einem Oberhaupt unterstanden. In der Mitte der Lager befand sich ein meterhoher Turm, von dem aus ein „Informationsminister“ tägliche Informationen und Anweisungen über Arbeitsregeln, Gemeinschaftsprojekte oder Sanktionen verlautbarte. Handwerkliche Goldminen in Burkina Faso sind extraktive Enklaven in dem Sinne, dass ihre Verwalter (früher eine staatliche Firma, heute Privatunternehmer) zumindest teilweise in der Lage sind, vor Ort von der Regierung unabhängige Machtstrukturen zu etablieren. ${ }^{18}$

Während Ferguson enclaving als charakteristischen Modus von RohstoffVerwaltung prognostiziert, steht in anderen Diskussionen über extraktive Aktivitäten die frontier im Vordergrund. In Diskursen über Bergbau ist die Enklave eine von mehreren wiederkehrenden Raummetaphern, die in den Kategorien des SFB 1199 „imaginierte Raumformate“ anzeigen können. Daneben tritt prominent auch die Metapher der frontier auf. Wenn etwa Shreshta und Smith den afrika-

15 Magrin, Voyage en Afrique rentière, S. 424.

16 B. Rubbers, „Mining Towns, Enclaves and Spaces: A Genealogy of Worker Camps in the Congolese Copperbelt“, Geoforum (i. E.).

17 J. Omasombo Tshonda, „Les diamants de Kisangani: de nouveaux seigneurs se taillent des fiefs sur le modèle de l'État zaïrois de Mobutu“, in: L. Monnier, B. Jewsiewicki und G. de Villers (Hg.), Chasse au diamant au Congo/Zaïre, Tervuren, Paris: Institut Africain/L'Harmattan, 2000, S. $79-126$.

18 K. Werthmann, „The President of the Gold Diggers: Sources of Power in a Gold Mine in Burkina Faso“, Ethnos 68 (2003) 1, S. 95-111; Dies., „The Drawbacks of Privatization: Artisanal Gold Mining in Burkina Faso 1986-2016“, Resources Policy 52 (2017), S. 418-426. 
nischen Kontinent als „last frontier of globalization“ für internationale Investoren bezeichnen, ${ }^{19}$ so verweisen sie auf vermeintlich unerschlossene Räume sowie auf den Unternehmergeist und die Risikobereitschaft, welche nach Frederick Jackson Turner ${ }^{20}$ charakteristisch für die europäischen Pioniere im amerikanischen Westen waren und den US-amerikanischen Nationalcharakter prägten. Historiker wie Turner sowie viele autobiographische und fiktionale Werke trugen zu einer Romantisierung der frontier bei, welche die heroische Erschließung neuer Räume symbolisiert. $^{21}$

Igor Kopytoff wandte das Konzept der frontier auf historische Prozesse im vorkolonialen Afrika an, wo sich in territorialen und politischen Zwischenräumen bzw. innerhalb eines ,institutionellen Vakuums“ neue Arten von politischer Organisation und Gesellschaft herausbilden konnten. ${ }^{22}$ Dumett verwendete die Bezeichnung frontier und „Eldorado“ in seiner Studie zum kolonialen Goldbergbau in Ghana. ${ }^{23}$ Er argumentiert, dass der Goldboom von 1877 bis 1897 durch die Ausbreitung von zwei parallelen frontiers geprägt war: einer afrikanischen und einer europäischen. Arbeiter im industriellen Bergbau führten neue Technologien in den bereits existierenden handwerklichen Bergbau ein, der dadurch konkurrenzfähig wurde.

Filip De Boeck spricht mit Bezug auf Diamantenfördergebiete in Angola davon, dass das „frontier setting“ der Schürfgebiete nicht nur eine spezifische Verräumlichung meine, sondern ein Geisteszustand (state of mind) sei, in dem lokale und globale Vorstellungswelten über Männlichkeit, Wohlstand und Konsum miteinander verschmelzen. ${ }^{24}$ Tilo Grätz beschreibt Goldfördergebiete in Westafrika als „frontiers of artisanal mining“, ${ }^{25}$ in denen lokale kleinbäuerliche

19 N. R. Shrestha und W. I. Smith „Plowing the Last Frontier of Globalization: Management Implications for Africa's Development“, in: S. Sigué (Hg.), Repositioning African Business and Development for the 21st Century, Proceedings of the 10th Annual Conference, IAABD, 2009.

20 F. J. Turner, The Frontier in American History, The Floating Press (Epub), 2014 [1893].

21 G. Pisarz-Ramirez, S. Wöll und D. Bozkurt, Spatial Fictions: Imagining (Trans)national Space in the Southern and Western Peripheries of the Nineteenth Century United States, Working paper series SFB 1199 No. 10 (2018).

22 I. Kopytoff, „The Internal African Frontier - the Making of African Political Culture“, in: I. Kopytoff (Hg.), The African Frontier. The Reproduction of Traditional African Societies, Bloomington/Indianapolis: Indiana University Press, 1987, S. 3-84.

23 Dumett, El Dorado in West Africa.

24 F. De Boeck, Filip, „Garimpeiro Worlds: Digging, Dying und ,Hunting for Diamonds in Angola“, Review of African Political Economy 28 (2001) 90, S. 548-562.

25 T. Grätz, „Les frontières de l’orpaillage en Afrique occidentale“, Autrepart 2 (2004) 30, S. 135150; Ders., The ,frontier' revisited: gold mining camps and mining communities in West Africa, ZMO Working Papers 10 (2013). 
Gemeinden und zugewanderte Schürfer neue Formen von Arbeit und sozialen Beziehungen erproben. ${ }^{26}$ Deborah Fahy Bryceson und Paul Yankson charakterisieren den handwerklichen Bergbau in Ghana und Tansania als „frontier livelihoods“, die mit einer „frontier mystique“ verbunden seien: das geologische und soziale Unbekannte werde mit enormem wirtschaftlichen Potential assoziiert. ${ }^{27}$ Gold- und Diamantenfrontiers sind darüber hinaus liminale Räume, in denen junge Männer einen initiationsähnlichen Übergang zum Erwachsenenstatus durchlaufen. ${ }^{28}$ Sie sind aber auch Zufluchtsorte für Männer und Frauen, die aus verschiedenen Gründen nicht in ihre Herkunftsgemeinschaften zurückkehren können oder wollen sowie Heterotopien für unkonventionelle Lebensweisen und Geschlechterverhältnisse. ${ }^{29}$ Wie in Kopytoffs Modell beziehen sich diese neuen Lebensformen auf vorhandene soziale Ordnungen und staatliche Regelungen, bringen aber auch Innovationen hervor, z. B. im Bereich der Risikoteilung.

Luigi Arnaldi di Balme und Cristiano Lanzano beschreiben einheimische Privatunternehmer im handwerklichen Goldbergbau von Burkina Faso als „frontier entrepreneurs“, die diesen Sektor im Zuge der Liberalisierung neu strukturieren. ${ }^{30}$ Bryceson et al. postulieren, dass Grubenbesitzer und Schürfer in Tansania aufgrund ihrer Erfahrungen mit Selbstorganisation, sozialer Heterogenität und flachen Hierarchien einen „frontier ethos“ teilen und daher tendenziell demokratischer eingestellt seien als andere Landsleute. ${ }^{31}$

In einer Fußnote erwähnte Frederick Jackson Turner auch Spieler, Desperados und Vigilanten als „gesetzeslose“ frontier-Typen. Diese Typen kehren in Morten

26 K. Werthmann und T. Grätz (Hg.), Mining Frontiers in Africa. Anthropological and Historical Perspectives, Köln: Köppe, 2012.

27 D. F. Bryceson und P. Yankson, „Frontier Mining Settlements: Livelihood Promises and Predicaments“, in: J. Agergaard, N. Fold und K. V. Gough (Hg.), Rural-Urban Dynamics: Livelihoods, Mobility and Markets in African and Asian Frontiers, London: Routledge, 2010, S. 189-197.

28 F. De Boeck, „Domesticating Diamonds and Dollars: Expenditure, Identity and Sharing in Southwestern Zaire“, Development and Change 29 (1998), S. 777-810.

29 K. Werthmann, Bitteres Gold. Bergbau, Land und Geld in Westafrika, Köln: Köppe, 2009; Dies., „Following the Hills: Gold Mining Camps as Heterotopias“, in: U. Freitag und A. von Oppen (Hg.), Translocality: The Study of Globalising Processes from a Southern Perspective, Leiden: Brill, 2010, S. 111-132.

30 L. Arnaldi di Balme und C. Lanzano, „'Entrepreneurs de la frontière‘: le rôle des comptoirs privés dans les sites d'extraction artisanale de l'or au Burkina Faso“, Politique africaine 3 (2013) 131, S. $27-49$.

31 D. F. Bryceson, E. Fisher, J. B. Jønsson und R. Mwaipopo (Hg.), Mining and Social Transformation in Africa: Mineralizing and Democratizing Trends in Artisanal Production, London: Routledge, 2014. 
Bøås’ Darstellung der borderlands wieder. ${ }^{32}$ Bøås bezieht sich auf „Konfliktökonomien“, die Schürfern, Kaufleuten und Kriegern ein geeignetes Umfeld für Aktivitäten bieten. Borderlands sind nicht vollständig vom Nationalstaat abgekoppelt, liegen oft aber außerhalb der Reichweite von Regierungen, insbesondere im Fall von Bürgerkriegsgebieten, die von Rebellengruppen kontrolliert werden. In diesen borderlands gedeihen informelle wirtschaftliche Aktivitäten wie handwerklicher Bergbau und Schmuggel.

Bøås’ Konzept von borderlands erinnert an Georg Elwerts „Gewaltmärkte“33 als „sich reproduzierende wirtschaftliche Systeme der Gewaltnutzung und Gewalterzeugung“34. „Gewaltmanager“ wie Kriegsherren verschaffen sich und ihrer Gefolgschaft ein Einkommen und können unter Umständen der betroffenen Bevölkerung relative Sicherheit bieten. Andere agieren als Gewaltherrscher, die die lokale Bevölkerung ausbeuten, vertreiben oder vernichten. Informelle Netzwerke und „twilight institutions“ ${ }^{35}$ können die borderlands mit Machtzentren verbinden. Dies ist der Fall beim Big Man, der politische Vernetzung und wirtschaftliche Potenz kombiniert. Er profitiert von einer Konfliktökonomie, da er Informationen von verschiedenen Seiten bezieht. Durch Redistribution, Überzeugung und Gewalt kann er Kapital und Gefolgschaft mobilisieren. ${ }^{36}$ Borderlands sind Möglichkeitsräume für unternehmerische und gut vernetzte Individuen, personifiziert im Big Man. Für viele andere sind sie Räume der Einschränkung und Unterdrückung durch Gewaltunternehmer.

Auch wenn frontiers und borderlands Ähnlichkeiten aufweisen, betont Michael Watts, dass die frontier als spezifischer sozialer Raum durch ihr Verhältnis zu Institutionen und Prozessen - insbesondere des Staates - bestimmt ist. ${ }^{37}$

32 M. Bøås, The Politics of Conflict Economies: Miners, Merchants and Warriors in the African Borderland, London: Routledge, 2015.

33 G. Elwert, „Markets of Violence“, in: G. Elwert, S. Feuchtwang und Dieter Neubert (Hg.), Dynamics of Violence. Processes of Escalation and De-Escalation in Violent Group Conflict (Sociologus Beiheft 1), Berlin: Duncker und Humblot, 1999, S. 85-102.

34 G. Elwert, „Wie ethnisch sind Bürgerkriege? Der Irrglaube, daß Bürgerkriege kulturelle Wurzeln haben“, E+Z Entwicklung und Zusammenarbeit 10 (1998), S. 265-267, hier S. 267.

35 C. Lund (Hg.), Twilight Institutions: Public Authority and Local Politics in Africa, Oxford: WileyBlackwell, 2007.

36 Werthmann, „The President of the Gold Diggers“; S. W. J. Luning, „Gold Mining in Sanmatenga, Burkina Faso: Governing Sites, Appropriating Wealth“, in: J. Abbink und A.v. Dokkum (Hg.), Dilemmas of Development: Conflicts of Interest and their Resolutions in Modernizing Africa, Leiden: African Studies Centre, 2008, 195-211; M. Utas, „Introduction: Bigmanity and Network Governance in Africa“, in: Ders. (Hg.), African Conflicts and Informal Power: Big Men and Networks, London: Zed Books, 2012, S. 1-34.

37 M. J. Watts, „Frontiers: Authority, Precarity, and Insurgency at the Edge of the State“, World Development 101 (2018), S. 477-488. 
Frontiers sind Räume, in welchen Formen von Herrschaft und Autorität instabil und umstritten sind. Wie er an zwei unterschiedlichen Beispielen aus Nigeria (Konflikte um Öl in Südnigeria, Boko Haram in Nordnigeria) zeigt, sind die Aufstände an diesen frontiers ein Ergebnis einer tiefen Krise von Autorität und Herrschaft sowie einer fundamentalen Prekärität der Lebensumstände, insbesondere für junge Männer. Beides ist wiederum ein Resultat des nigerianischen resource curse.

In den hier aufgeführten Verwendungen ist die frontier sowohl ein konkreter als auch ein imaginierter Raum, der von Vorstellungen über ein anderes und besseres Leben geprägt wird. Dabei besteht wie an der historischen frontier in den USA des 19. und frühen 20. Jahrhunderts die Tendenz, die bereits dort lebenden Menschen und deren Landnutzungsformen zu übersehen oder für irrelevant $\mathrm{zu}$ erklären. ${ }^{38}$ Der Bezug auf die frontier des historischen amerikanischen Westens enthält aber auch die Gefahren, die ihr innewohnen, sowie die mit der Erschließung verbundene Gewalt gegen andere. Das Konzept der frontier ermöglicht es, diese ambivalenten Aspekte der Rohstoff-Förderung zu erfassen.

Es fällt auf, dass die beiden Metaphern der Enklave und der frontier in einem engen inhaltlichen Zusammenhang auftreten und in bestimmten Diskursen als geeignet angesehen werden, gemeinsam eine Transformation der Raumordnung zu beschreiben. Dies kann ein Indikator dafür sein, dass Enklave und frontier auch in der Praxis des Verräumlichens einen direkten Zusammenhang bilden. In der historischen Analyse von Verräumlichungsprozessen während der Herausbildung der extraktraktiven Industrie im kolonialen Tansania zeigt sich eine enge Verwobenheit von frontier und Enklave. Frontiers, welche oft als „leere“ oder „herrenlose“ Gebiete angesehen werden, bieten dabei den Rahmen für Territorialisierungsprozesse seitens (kolonialer) Bergbauunternehmen oder individueller Akteure, welche durch den Erwerb staatlicher Lizenzen formalisiert und legitimiert werden. Die im vermeintlich herrenlosen Terrain produzierten Territorien, oft fernab des Firmensitzes oder des effektiven Einflussbereiches der Kolonialregierung, sehen sich jedoch mit der Territorialität anderer Akteure wie lokaler Bevölkerungsgruppen konfrontiert. Die Intensivierung territorialer Praktiken seitens der Unternehmen oder anderer Akteursgruppen kann zu enclaving führen, da die Abschottung und Sicherung des eigenen, zur Ressourcenförderung vorgesehenen, Territoriums gegenüber Einflüssen von außen angestrebt wird. Dies geschieht auch mit Unterstützung des kolonialen Staates, beziehungsweise der

38 S. W. J. Luning, „Mining temporalities: Future perspectives“, The Extractive Industries and Society 5 (2018), S. 281-286. 
Unternehmen und Muttergesellschaften. Auf diese Weise produzierte Enklaven stellen Inseln der Stabilität inmitten fragiler Umgebungen dar. ${ }^{39}$

Die skizzierte Beziehung zwischen frontier und Enklave lässt sich auch in rezenten Ressourcenbooms nachvollziehen. Côte und Korf sehen Konzessionsgebiete für industrielle Goldproduzenten in Burkina Faso als frontiers, weil dort gleichzeitig andere frontier entrepreneurs wie handwerkliche Goldschürfer und Lokalpolitiker um die Regulierung der Förderung ringen: „Such concession gives rise to a form of entangled capitalism that profits both frontier entrepreneurs, the exploration concessionary and the orpaillage miners, and it is an integral part of the conditions under which extractive enclaves emerge“ “. ${ }^{40}$

Auch in unserer eigenen Forschung zum Goldbergbau in Burkina Faso und Tansania konnten wir beobachten, dass Tendenzen zur Enklavenbildung im industriellen Bergbau begrenzt oder verhindert werden durch andere Organisationen und Institutionen. Das ist zum einen die artisanal mining frontier, aber auch andere Akteure um Umfeld eines Bergbauprojektes. Anders als es Fergusons Bild vom „hüpfenden“ Kapital suggeriert, welches seine Berührung mit dem betreffenden Staat auf ein Minimum reduziert, müssen sich multinationale Bergbauunternehmen heute zumindest symbolisch transnationalen und nationalen Regulierungsinstanzen und -formen anpassen und/oder vor Ort mit zunehmend selbstbewussten Lokalregierungen und zivilgesellschaftlichen Organisationen interagieren.

In Tansania versucht die Regierung, eine Enklavenbildung durch Betonung nationalstaatlicher Souveränität einzuschränken. Mit Maßnahmen wie Exportverboten, Beschlagnahmung von Erzkonzentraten, neuen Gesetzen sowie schwerwiegenden Anschuldigungen, welche Betrug und Steuerhinterziehung beinhalten, zwang die Regierung den größten Goldproduzenten des Landes Acacia Mining an den Verhandlungstisch. Die Gesetzesänderungen sehen unter anderem eine stärkere Rolle des Staates in der Regulierung der Rohstoffförderung vor, etwa durch direktes Engagement in Joint Ventures oder die Akquise von Unternehmensanteilen. Abgesehen von einer Radikalisierung des bereits seit einiger Zeit bestehenden Ressourcennationalismus mithilfe national-populistischer Rhetorik sind auch neo-extraktivistische Tendenzen in Tansania vorzufinden, welche für den Staat nicht nur die Rolle des Empfängers von Rohstoffrenten vorsehen, sondern des zentralen Akteurs in der Durchführung und Kontrolle

39 Hönke, „Extractive Orders“.

40 Côte und Korf, „Making Concessions“, S. 472. 
extraktiver Praktiken und in der Verteilung der Profite. Dies wird auch als Rückkehr des „Entwicklungsstaates“ gedeutet. ${ }^{41}$

In Tansania versucht der Staat, direkteren Zugriff auf die Förderstätten der multinationalen Konzerne zu erhalten. Die Unternehmen versuchen dies zu verhindern, indem beispielsweise Regierungsbeamte am Betreten von Minengeländen gehindert werden. 2018 ließ Präsident Magufuli durch das Militär eine Mauer um die Tansanit-Minen im Norden des Landes errichten, um dem Raubbau durch Unbefugte vorzubeugen und die staatliche Kontrolle über Förderung und Export zu erhöhen. Weiterhin gab es im Rahmen der Verhandlungen mit Acacia Mining Bestrebungen, die stark durch eine Betonung nationalstaatlicher Territorialität gekennzeichnet waren. So verlangte die tansanische Regierung die Verlegung des Firmensitzes und der Firmenkonten von Acacia nach Tansania.

Historisch betrachtet zeigt sich in Tansania ein Zickzack von „sovereignty regimes“. Die aktuell beobachtbaren Tendenzen erinnern deutlich an die sozialistische Ära Tansanias (1961-1985) unter Julius Nyerere, während welcher der Entwicklungsstaat als zentraler, regulierender Akteur vollständige territoriale Souveränität anstrebte. Im Kontrast dazu standen die koloniale Periode (18851961) sowie die Zeit der Liberalisierung (seit 1985), welche durch geringe staatliche Kontrolle oder aktive Unterstützung eine Enklavenbildung begünstigten. Folglich zeigt sich am Beispiel des Bergbaus in Tansania, dass Enklavenbildung kein rezentes Phänomen ist, sondern auch historisch von Aushandlungen zwischen staatlichen und nicht-staatlichen Akteuren geprägt war. Der Nationalstaat verliert im Zug der Globalisierung nicht unbedingt seine Relevanz, sondern kann, wie z. B. auch in Lateinamerika, eine stärkere Rolle als Hauptakteur für eine ressourcenbasierte Entwicklungspolitik (Neoextraktivismus) anstreben. ${ }^{42}$

In Burkina Faso sind es neben der Regierung auch Akteure auf anderen Ebenen, die Tendenzen zur Enklavenbildung entgegentreten. ${ }^{43}$ Die derzeitige Situation in Burkina Faso ist vom politischen Umbruch infolge des Volksaufstandes 2014 und der Neuwahlen 2015 geprägt. Das Regime von Blaise Compaoré (19872014) war u.a. wegen der Korruption im Bergbausektor unter Druck geraten. Burkina Faso ist Mitgliedsstaat der Regionalorganisationen Economic Community of West African States (ECOWAS) und der West African Economic and Monetary

41 T. Jacob und R. H. Pedersen, „New Resource Nationalism? Continuity and Change in Tanzania's Extractive Industries, The Extractive Industries and Society 5 (2018) 2, S. 287-292; K. Roder, „,Bulldozer Politics‘. State-Making and (Neo-)Extractive Industries in Tanzania’s Gold Mining Sector“, The Extractive Industries and Society 6 (2019), S. 407-412.

42 Ebd.

43 K. Werthmann und D. Ayeh, Processes of Enclaving under the Global Condition: The Case of Burkina Faso, SFB 1199 Working Papers 4 (2017); Werthmann, „The Drawbacks of Privatization“. 
Union (WAEMU). WAEMU formulierte 2003 einen Common Mining Code, an dem sich die Mitgliedsländer orientieren sollten. ECOWAS erließ im Jahr 2009 die Directive on the Harmonization of Guiding Principles and Policies in the Mining Sector. Die ECOWAS-Direktive enthält u. a. die Bestimmung, dass Firmen von den örtlichen Gemeinschaften freie, vorherige und informierte Zustimmung erhalten müssen, bevor die Erkundungsarbeiten beginnen können sowie vor jeder nachfolgenden Phase von Bergbau- und Bergbaufolgeaktivitäten. Diese Vorgaben sollen einerseits die Attraktivität westafrikanischer Länder für ausländische Investoren erhöhen, andererseits den Staaten eine größere Handhabe bezüglich der Gewinne aus dem Bergbau und ihrer Verwendung ermöglichen. In Burkina Faso trat 2015 ein reformiertes Bergbaugesetz in Kraft, welches Gebühren, Steuern und Abgaben für multinationale Unternehmen neu regelt. Bergbau-Unternehmen sollen nun höhere Gebühren, Steuern und Abgaben entrichten.

In den Anrainergemeinden von Bergbauprojekten sind zunehmend zivilgesellschaftliche Organisationen und Bündnisse aktiv. Hinzu kommen spontane Zusammenschlüsse von Jugendlichen oder von durch Bergbau von Enteignung Betroffenen, die gegenüber multinationalen Unternehmen ihre Rechte auf Zugang zu Land, Wasser und Anbauflächen einfordern. Proteste können mit Vandalismus und der Zerstörung von (Minen-)Infrastruktur einhergehen. Ihren „Anteil am Gewinn“ fordern Protestbewegungen dabei nicht nur von den Unternehmen selbst ein, sondern auch vom Staat als Verwaltungs- und Verteilungsinstanz der Bergbaurente oder von lokalen Bündnispartnern der Unternehmen. ${ }^{44}$

Dass in Burkina Faso Aushandlungen mit Unternehmen heute auch in den Anrainergemeinden der Bergbauprojekte stattfinden, steht u.a. im Zusammenhang mit dem seit 1998 in Gang gesetzten Dezentralisierungsprozess und den ersten landesweiten Kommunalwahlen 2006. In Bezug auf Bergbaurechte ist hier vor allem die im Bergbaugesetz von 2015 vorgesehene Einrichtung eines „Bergbaufonds für lokale Entwicklung“ bedeutend, welcher durch die lokalen Gebietskörperschaften (Kommunen) verwaltet werden soll, bislang aber noch nicht eingerichtet wurde. Der Fonds könnte insofern entscheidend zu einem weiteren de-enclaving des burkinischen Bergbausektors beitragen, als dass auch lokale Gebietskörperschaften von der nationalen Bergbaurente profitieren würden. Bisher sind von Bergbau betroffene Gemeinden vor allem vom „goodwill“ der Unternehmen abhängig, welche ethische Leitlinien und Programme wie z. B. im

44 Ayeh o. J., mining-conflicts-burkina.net. 
Rahmen der Extractive Industries Transparency Initiative mehr oder weniger konsequent umsetzen, um dadurch eine „social license to operate“ ${ }^{\text {45 }} \mathrm{zu}$ erwerben.

Im Zuge von extraktiven Aktivitäten tritt Enklavenbildung nicht nur in Konkurrenz zum Nationalstaat auf, sondern auch zur Aneignung und Verwaltung natürlicher Ressourcen durch Akteure auf lokaler Ebene. Dies sind beispielsweise handwerkliche Schürfer, aber auch Anwohner der Bergbaugebiete, die keinen Zugang zu Entschädigungszahlungen oder zu Beschäftigungen im Bergbau erhalten. In den Aushandlungen oder Kämpfen über die Instanzen und Mechanismen, die den Zugang zu natürlichen Ressourcen regeln, wird jedoch deutlich, dass Akteure sich keineswegs immer mit einer Stimme gegenüber multinationalen Unternehmen positionieren. Es existieren vielmehr unterschiedliche Vorstellungen darüber, welche Räume und Akteure wie vom Rohstoffboom profitieren sollten und welche nicht.

Vor Ort schließen Akteure strategische Bündnisse und vernetzen sich auf verschiedenen Ebenen. So kommt es beispielsweise im Zuge von lokalen Protesten gegen multinationale Unternehmen zur Mobilisierung einer nationalen bzw. internationalen Öffentlichkeit. ${ }^{46}$ In Burkina Faso sind in mehreren vom Bergbau betroffenen Gemeinden Ortsgruppen der linksgerichteten Organisation démocratique de la jeunesse (ODJ) aktiv und unterstützen die lokale Bevölkerung bei der Formulierung und medialen Verbreitung von Forderungskatalogen oder der Organisation von Protestaktionen. Angehörige der ODJ beteiligten sich in Houndé im Westen des Landes 2016 an einer Exkursion einer lokalen Vereinigung von Gewerbetreibenden und Jugendlichen nach Ouahigouya in einem nördlichen Landesteil. In beiden Orten betreibt das multinationale Unternehmen Endeavour industrielle Goldminen. Durch die Exkursion sollten die Teilnehmenden aus Houndé Informationen über die teilweise erfolgreichen Proteste und Verhandlungen in Ouahigouya erhalten als Grundlage für die weitere Planung von Aktivitäten in Houndé. Mitglieder der ODJ sind wiederum international vernetzt und repräsentieren die Organisation z. B. auf Veranstaltungen in Europa.

Eine andere im ganzen Land aktive zivilgesellschaftliche Organisation ist die Organisation pour le Renforcement des Capacités de Développement (ORCADE). Sie organisiert $u$. a. Veranstaltungen für Bürger und Vertreter von Gebietsköperschaften, um diese über das neue Bergbaugesetz zu informieren. Auf dieser Grundlage können vom Bergbau Betroffene ihre Anliegen formulieren, die sie an

45 J. Gehman, L. M. Lefsrud und S. Fast, „Social License to Operate: Legitimacy by Another Name?“ Canadian Public Administration 60 (2017) 2, S. 293-317.

46 B. Engels, „Wann werden Konflikte manifest? Politische Opportunitätsstrukturen für Proteste gegen Goldbergbau in Burkina Faso“, Peripherie 37 (2017), S. 146-147, 297-318. 
Bürgermeister, Abgeordnete der Nationalversammlung und Unternehmen addressieren.

Enklaven und frontiers sind als imaginierte Raumformate in wissenschaftlichen und populären Diskursen über Rohstoffförderung relevant. Die frontier wird mit neuen Horizonten und unerschlossenen Ressourcen assoziiert und bietet einen Möglichkeitsraum oder Legitimationsgrund für Unternehmungen verschiedener Art. Während die Rede von der frontier eher Offenheit und Chancen suggeriert, werden Enklaven mit Intransparenz und Einschränkungen verbunden. Ein Fördergebiet für Rohstoffe kann beides zugleich sein: aus der Sicht von Unternehmen eine „last frontier of globalization“, aus der Sicht betroffener Bevölkerungen eine Enklave (,andere Welt“, „Staat im Staate“ o. a.). 